\title{
Liraglutide ameliorates nonalcoholic fatty liver disease in diabetic mice via the IRS2/PI3K/Akt signaling pathway
}

This article was published in the following Dove Press journal: Diabetes, Metabolic Syndrome and Obesity: Targets and Therapy

\author{
Pijian Yang ${ }^{1} *$ \\ Yuzhen Liang ${ }^{2, *}$ \\ Yunchen Luo ${ }^{2}$ \\ Zhengming $\mathrm{Li}^{2}$ \\ Yumei Wen' \\ Jing Shen' \\ Ruwen $\mathrm{Li}^{1}$ \\ Hua Zheng ${ }^{3}$ \\ Harvest $\mathrm{F} \mathrm{Gu}^{4, *}$ \\ Ning $X_{i a}{ }^{l, *}$ \\ 'Department of Endocrinology and \\ Metabolism, The First Affiliated Hospital \\ of Guangxi Medical University, Nanning \\ 53002I, People's Republic of China; \\ ${ }^{2}$ Department of Endocrinology and \\ Metabolism, The Second Affiliated \\ Hospital of Guangxi Medical University, \\ Nanning 53002I, People's Republic of \\ China; ${ }^{3}$ Life Sciences Institute, Guangxi \\ Medical University, Nanning 53002I, \\ People's Republic of China; ${ }^{4}$ Center for \\ Pathophysiology, School of Basic \\ Medicine and Clinical Pharmacy, China \\ Pharmaceutical University, Nanjing \\ 210009 People's Republic of China \\ *These authors contributed equally to \\ this work
}

Correspondence: Ning Xia Department of Endocrinology and Metabolism, The First Affiliated Hospital of Guangxi Medical University, Nanning 53002I, People's Republic of China Tel +86 77I 2802380

Email xianinggxmu@I63.com
Purpose: High prevalence of nonalcoholic fatty liver disease (NAFLD) among patients with type 2 diabetes has implicated the role of hepatic insulin resistance (IR) in the diseases. To better understand the underlying mechanism, we have evaluated the pathophysiological effects of Liraglutide on NAFLD via the insulin signaling pathway.

Patients and methods: A $2 \times 2$ factorial experiment was designed. High-fat diet (HFD)-induced NAFLD mice with diabetes were treated with Liraglutide for 10 weeks, while the control mice were saline-treated. Hepatic expressions of InsR, IGF-1R, IRS2, PI3K and Akt at mRNA and protein levels were analyzed with RT-PCR and Western blotting. Hematoxylin and eosin staining, Oil Red O staining and electron microscopy were used to visualize triglyceride accumulation in liver.

Results: Liraglutide significantly decreased body weight, fasting blood glucose levels and HOMA-IR scores in HFD mice. Compared with the control mice fed with chow diet, hepatic expressions of InsR, IRS2, PI3K and Akt at both mRNA and protein levels in HFD mice were significantly reduced, but upregulated after Liraglutide treatment. Furthermore, Liraglutide treatment was found to improve hepatic steatosis.

Conclusion: The current study thereby provides evidence that Liraglutide ameliorates NAFLD and improves hepatic steatosis mainly by upregulation of the IRS2/PI3K/Akt signaling mediators.

Keywords: glucagon like peptide 1, Liraglutide, nonalcoholic fatty liver disease, insulin resistance, insulin signaling

\section{Introduction}

Nonalcoholic fatty liver disease (NAFLD) is defined as a manifestation of fat accumulation in liver but not caused by excessive alcohol drinking. In pathophysiology, NAFLD can be divided into nonalcoholic fatty liver, nonalcoholic steatohepatitis, hepatic fibrosis and hepatic cirrhosis. ${ }^{1}$ In recent years, the prevalence of NAFLD among patients with type 2 diabetes (T2D) has been found to be increased up to $70 \% .^{2}$ There is a closed correlation between NAFLD and T2D because NAFLD increases the risk of incident T2D, while T2D also contributes to the progression of NAFLD. ${ }^{3}$ It has been demonstrated that chronic liver disease is mostly attributed to NAFLD in patients with T2D. ${ }^{4}$ Subjects with diabetes, however, have an increased risk of developing cirrhosis and liver failure. ${ }^{5}$ This risk increases the urgency of developing pharmacological therapies. Therefore, it is of importance to clarify the detailed mechanisms of NAFLD progression in diabetes. 
Glucagon-like peptide-1 (GLP-1) is secreted by intestinal L-cells and is produced through the posttranslational processing of proglucagon in response to nutrient ingestion. ${ }^{6}$ Recently, GLP-1 receptors are believed to be expressed on human hepatocytes. ${ }^{7}$ Liraglutide is a longacting GLP-1 analog with 97\% homology to human GLP1 and is used as a long-acting GLP-1 receptor agonist. ${ }^{8}$ Recent studies have shown that Liraglutide can improve hepatic steatosis in a weight loss-independent manner. ${ }^{9}$ Although Liraglutide exhibits positive effects on hepatic steatosis, its definitive mechanism is still unclear.

Insulin resistance (IR) is the common pathophysiological basis of T2D and NAFLD and promotes the accumulation of fat in hepatocytes by promoting lipolysis and hyperinsulinemia. ${ }^{10,11}$ Since IR is believed to be the main cause of NAFLD, the drugs targeting IR may be used for the treatment of NAFLD. ${ }^{12}$ Liraglutide was primarily used to treat T2D, which was proven to be effective in alleviating IR. Abnormalities in the insulin signaling pathway play a key role in the occurrence and development of IR. Insulin receptor substrates (IRSs) are important cytoplasmic adaptor proteins in insulin signaling and play an important role in IR. ${ }^{13}$ The IRS family comprises several members, including IRS-1, IRS-2, IRS-3, IRS-4, IRS-5 and IRS-6. ${ }^{14}$ Among them, IRS-2 in liver specifically regulates insulin signaling and integrates insulin receptor (InsR) and insulin-like growth factor-1 receptor (IGF1R) signaling, which mediates the anabolic effects of insulin through the PI3 kinase (PI3K)-Akt cascade. ${ }^{15,16}$ Furthermore, mice lacking IRS-2 has shown a defective insulin-stimulated signaling pathway, which led to hepatic IR. ${ }^{17}$ Recent studies have shown that Liraglutide essentially restores brain insulin sensitivity through insulin signaling pathway. ${ }^{18,19}$ However, the specific association between Liraglutide and the insulin signaling pathway in hepatocytes has not yet been investigated.

In the current study, we have established a mouse model of NAFLD with T2D by using high-fat diet (HFD) feeding. With this mouse model, we have investigated the molecular mechanisms for Liraglutide to regulate the insulin signaling pathway in term of prevention of hepatic steatosis. Data from this study may provide useful information for better understanding the pathophysiological effects of Liraglutide on NAFLD via the insulin signaling pathway.

\section{Material and methods}

\section{Animal models}

C57BL/6J mice (5 weeks old, male) were purchased from Animal Experiment Center of Guangxi Medical University
(Nanning, People's Republic of China). The experimental mice were maintained in a specific pathogen-free room with a 12-hr light/dark cycle. In the first week, all mice were fed with a normal rodent chow diet $(5 \%$ fat $w t / w t)$. After then, the mice were randomly divided into two groups, ie, NC group: the mice fed with a normal chow diet $(5 \%$ fat $w t / w t)$ and HFD group: the mice fed with $\operatorname{HFD}(60 \%$ fat $\mathrm{wt} / \mathrm{wt}$, Ready Biotechnology Co., Ltd. Shenzhen, People's Republic of China). Body weight (BW) and fasting blood glucose (FBG) levels were monitored weekly. In HFD group, the mice with FBG levels $>13.9 \mathrm{mmol} \mathrm{L}^{-1}\left(250 \mathrm{mg} \mathrm{dL}{ }^{-1}\right)$ for 3 consecutive days were considered to be diabetic, and the mice were considered obese when their BW exceeded normal weight by at least $20 \%{ }^{20}$ After 10 weeks of chow diet or HFD feeding, the diabesity mice and obese were divided into two groups: a normal saline-treated group $(\mathrm{O}+\mathrm{S})$ and a Liraglutide-treated group $(\mathrm{O}+\mathrm{L})$. The control mice were also divided into two groups: a normal saline-treated group $(\mathrm{N}+\mathrm{S})$ and a Liraglutide-treated group $(\mathrm{N}+\mathrm{L})$. The mice were treated with a daily subcutaneous injection of Liraglutide $\left(0.2 \mathrm{mg} \mathrm{kg}^{-1}\right)$ or normal saline for 10 weeks. At the end of treatment, all mice were fasted for $8 \mathrm{hrs}$, anesthetized and sacrificed for blood and tissue collection. All experiments were approved by the Animal Ethics Committee of Guangxi Medical University, People's Republic of China. All experiments were conducted in accordance with the approved guidelines (the National Standard GB/T35892-2018 of the People's Republic of China). All efforts were made to minimize the suffering of mice (eg, the minimum concentration of drugs and pentobarbital sodium were applied) and the minimum number of mice were used to meet the valid statistical evaluation based on the guidelines of the animal ethics committee of the institute.

\section{Measurements of serum insulin, alanine aminotransferase (ALT), aspartame aminotransferase (AST) and blood glucose levels}

A mouse insulin ELISA kit (Cuabio Technology LLC, Wuhan, People's Republic of China) was used to measure serum insulin levels. A glucose meter (Johnson \& Johnson, New Brunswick, NJ, USA) was used to measure blood glucose levels. The HOMA-IR was used to assess IR. The HOMA-IR score was calculated as [fasting insulin $\left(\mathrm{mU} 1^{-1}\right) \times$ fasting glucose $\left.\left(\mathrm{mmol} \mathrm{l}^{-1}\right)\right] / 22.5 .^{20}$ Serum 
alanine ALT and aspartame aminotransferase AST were assayed by the enzyme method using commercially available kits (Wako Pure Chemical Industries, Ltd) with an Automated Biochemistry Analyzer 7100 (Hitachi, Ltd).

\section{Hematoxylin and eosin staining}

Fresh liver tissues were collected and post-fixed in $4 \%$ neutral buffered formalin solution for $24 \mathrm{hrs}$ followed by dehydration. The blocks were then trimmed, processed, and embedded in paraffin. The tissue slides were sliced at a thickness of $5 \mu \mathrm{m}$ and processed with HE staining.

\section{Oil red $O$ staining}

Oil Red $\mathrm{O}$ staining was used to visualize triglyceride accumulation in liver. Images were captured with a light microscope (Olympus, Tokyo, Japan).

\section{Masson staining}

Masson staining was used to visualize fibrosis in liver. Images were captured with a light microscope (Olympus, Tokyo, Japan).

\section{Ultrastructural changes of liver tissues}

Fresh liver tissues were collected, cut into sections of approximately $1 \mathrm{~mm} \times 1 \mathrm{~mm} \times 1 \mathrm{~mm}$, and fixed with $4 \%$ glutaraldehyde. After three rinses in $0.1 \mathrm{~mol} \mathrm{~L}^{-1} \mathrm{PBS}$, the specimens were dehydrated in an ascending ethanol series $(70 \%, 80 \%, 90 \%, 96 \%$ and $100 \%)$ followed by acetone $(100 \%$, water-free). The blocks were then trimmed, processed, and embedded in resin. Ultrathin sections $(60$ $\mathrm{nm}$ ) were cut using an ultramicrotome (Leica EM UC7, Leica Microsystems, Wetzlar, Germany). These sections were used for examination with light microscopy and also electron microscopy (EM, Hitachi H-7650 transmission electron microscope). Images were captured with a VELETA digital camera using Olympus Soft Imaging Solutions.

\section{Quantitative real-time RT-PCR analysis}

Total RNAs in liver were extracted using a TaKaRa MiniBEST Universal RNA Extraction Kit (Takara Bio, Shiga, Japan) according to the manufacturer's protocol, and cDNA was synthesized using PrimeScript RT Master Mix (Perfect Real Time) (Takara Bio, Shiga, Japan). The primers (Invitrogen, Shanghai, People's Republic of China) included the following: $\alpha$ - smooth muscle actin ( $\alpha$-SMA): forward primer (5'- TGCTGGACTCTGGAGATGGTGTG-3') and reverse primer (5'- CGGCAGTAGTCACGAAGGAATA-
GC-3'); InsR: forward primer (5'-GACAGCCACCACACTCACACTTC-3') and reverse primer (5'-GTGCAGCTCCTCATCACCATATCG-3'); IGF-1R: forward primer (5'-TGACATCCGCAACGACTATCA-3') and reverse primer (5'-CCAGTGCGTAGTTGTAGAAGAGT-3'); IRS2: forward primer (5'-TCTTTCACGACTGTGGCTTCCTT-3') and reverse primer (5'-CACTGGAGCTTTGCCCTCTGC-3'); PI3K p85: forward primer (5'-TTCCCTCGCAATAGGTTCTCC-3') and reverse primer (5'-GACCAATACTTGATGTGGCTGAC-3'); Akt: forward primer (5'-CATGAGGATCAGCTCGAACAGC-3') and reverse primer (5'ACGGGCACATCAAGATAACGG-3'); and $\beta$-actin: forward primer (5'-AAGAAGGTGGTGAAGCAGG-3') and reverse primer (5'-GAAGGTGGAAGAGTGGGAGT-3'). $\quad \beta$-Actin mRNA was used as the internal control. Quantitative realtime PCR was performed using FastStart Universal SYBR Green Master (ROX) (Roche Diagnostics, Indianapolis, IN, USA) on an Applied Biosystems StepOnePlus Real-Time PCR System (Thermo Fisher Scientific, Foster City, CA, USA). Gene expression was presented as the relative expression calculated using the $2^{-\Delta \Delta \mathrm{Ct}}$ method.

\section{Western blot}

Proteins were extracted from liver tissues by using a solution of PMSF and RIPA buffer. BCA protein quantification kit (Thermo Scientific) was used to measure the protein concentration. Western blotting was performed as previously described. ${ }^{20}$ The primary antibodies included antibodies against the $\alpha$-SMA (14395-1-AP; Proteintech, USA), IGF1 receptor+InsR (ab172965; Abcam, Cambridge, UK), $\beta$-actin (\#4970; Cell Signaling Technology, Danvers, MA), IRS-2 (\#4502; Cell Signaling Technology), PI3K p85 (\#4257; Cell Signaling Technology), phospho-PI3K p85 (\#4228; Cell Signaling Technology), Akt (\#4691; Cell Signaling Technology) and phospho-Akt (Ser473) (\#4060; Cell Signaling Technology). Goat anti-rabbit IgG H\&L (HRP) (ab205718; Abcam) was used as the secondary antibody. The membranes were scanned with an enhanced chemiluminescence system (ProteinSimple, Santa Clara, CA, USA).

\section{Statistical analysis}

All data are presented as the means and standard deviations (mean \pm SDs). Student's $t$-test, one-way ANOVA, a factorial analysis or Pearson's correlation was used to analyze significant differences, where appropriate. The homogeneity of variance test was used before Student's $t$-test or one-way 
ANOVA. $p<0.05$ was accepted as statistically significant. All analyses were conducted using SPSS 24.0 (SPSS Inc., Chicago, IL, USA).

\section{Results}

\section{Liraglutide improves metabolism in HFD-induced NAFLD mouse model}

Compared with NC group, mice in HFD group demonstrated significantly increased BW and FBG $(p<0.05)$ after 10 weeks of HFD feeding (Figure 1A and B). Histopathological examination in liver of HFD mice with HE staining exhibited hepatocyte ballooning, elevated steatosis, rarefaction of hepatocytic cytoplasm and clumped strands of intermediate filaments (Figures 1D and 4I). Histological sections of liver tissues in the mice after 10 weeks of normal rodent chow diet were represented in Figure 1C, which displayed normal liver architectures. Furthermore, the cellular lipid droplets in liver of HFD mice were visualized with Oil Red $\mathrm{O}$ staining and transmission electron microscopy (Figure 4J and K). Taking together, the data implied that the model of NAFLD with T2D was established.

NAFLD mice with T2D were then treated with a daily subcutaneous injection of Liraglutide or saline for 10 weeks. After treatment with Liraglutide, BWs and FBG levels in $\mathrm{O}+\mathrm{L}$ groups were significantly decreased (Figure 2A and B). Further factorial analyses revealed that there were significant differences in BWs, FBG levels, HOMA-IR scores, ALT and AST between NC and HFD mice and also between Liraglutide-treated and saline-treated groups Figure $3 \mathrm{~A}-\mathrm{E}$ ).

\section{Liraglutide reduces intrahepatocellular lipid accumulation}

We used $\mathrm{HE}$ and Masson staining to investigate the histopathological changes in the liver and used Oil Red $\mathrm{O}$ staining and transmission electron microscopy to visualize lipid droplets (Figure 4). The $\mathrm{N}+\mathrm{S}$ and $\mathrm{N}+\mathrm{L}$ groups displayed normal liver architectures. The normal structure of hepatic lobules in mice in the $\mathrm{O}+\mathrm{S}$ group was characterized by massive fatty degeneration. In contrast, a significant decrease in the severity of fat accumulation was observed in the liver tissues of mice in the $\mathrm{O}+\mathrm{L}$ group. Lipid droplets and fibrosis were undetectable in the livers of mice in the $\mathrm{N}+\mathrm{S}$ and $\mathrm{N}+\mathrm{L}$ groups but could be seen in the livers of mice in the $\mathrm{O}+\mathrm{S}$ group. Conversely, the livers of mice in the $\mathrm{O}+\mathrm{L}$ group exhibited a significant decrease in lipid droplet accumulation, and the fibrosis disappeared.
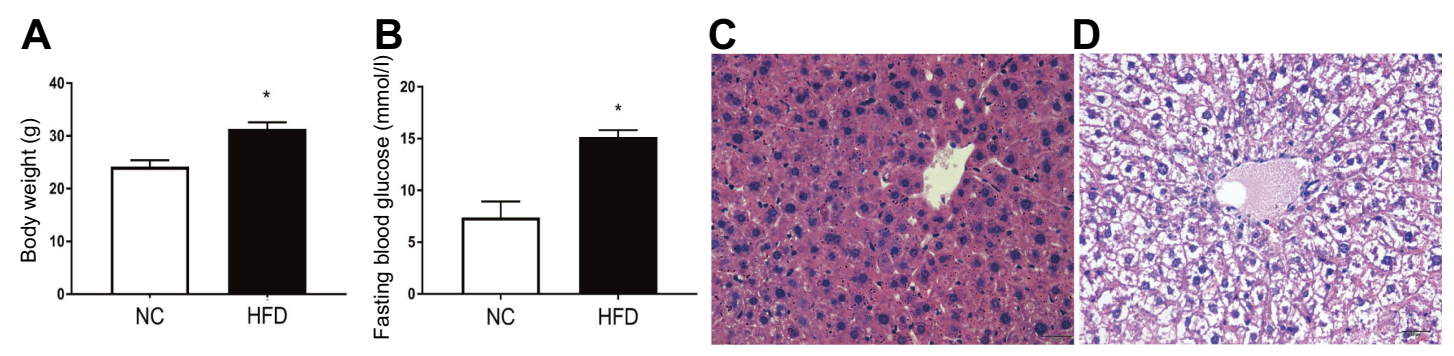

Figure I Physiological and histological evaluation of HFD-induced NAFLD mice. Body weight (A) and fasting blood glucose (B) levels in HFD-induced NAFLD mice without Liraglutide treatment (mean $\pm S D, n=12$ ). Histological sections of liver tissues in the mice after 10 weeks of normal rodent chow diet and HFD feeding were represented in (C and D) (stained with HE, and $\times 400$ ). ${ }^{*} p<0.05$ for comparison between NC and HFD groups.

Abbreviations: HFD, high-fat diet; NAFLD, nonalcoholic fatty liver disease; HE, hematoxylin and eosin staining; NC, the mice fed with a normal chow diet.
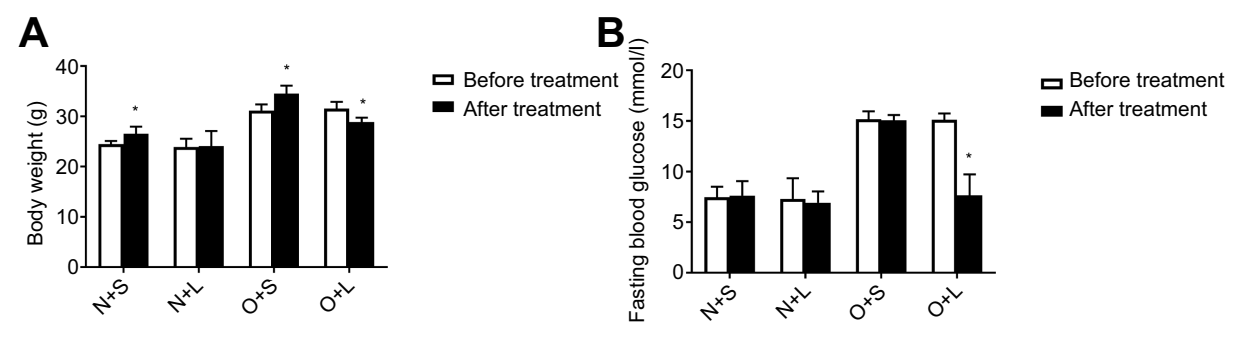

Figure 2 Body weight and fasting blood glucose levels in $N+S, N+L, O+S$ and $O+L$ groups before and after Liraglutide $(L)$ or saline $(S)$ treatment. Data were mean $\pm S D(n=6$ each group). (A) BW; (B) FBG level. * $p<0.05$ for before and after treatment.

Abbreviations: $\mathrm{N}+\mathrm{S}$, a normal saline-treated group; $\mathrm{N}+\mathrm{L}$, a Liraglutide-treated group; O+S, a normal saline-treated group; O+L, a Liraglutide-treated group; BW, body weight; FBG, fasting blood glucose. 

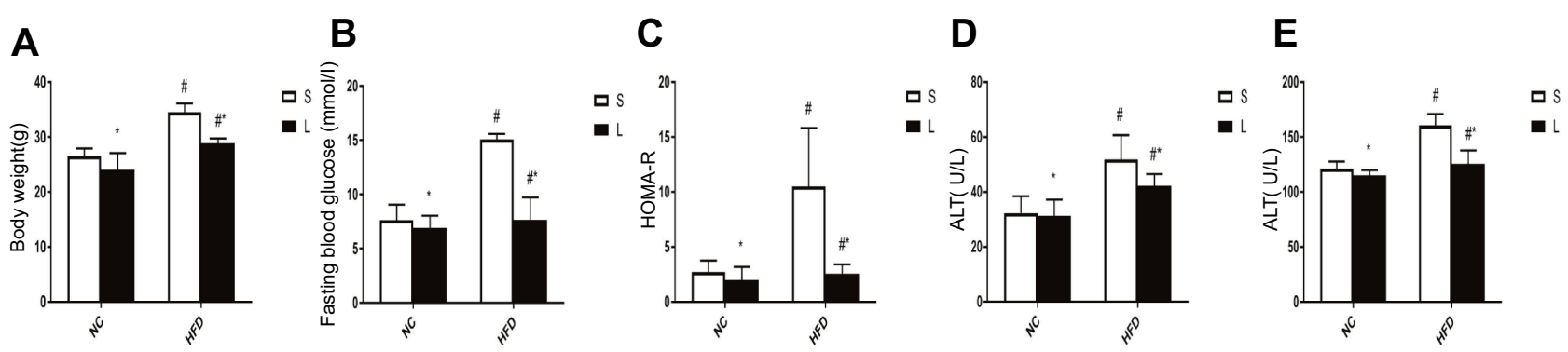

Figure 3 Body weight, fasting blood glucose levels, HOMA-IR scores, ALT and AST in mice after Liraglutide (L) or saline (S) treatment. Data were mean \pm SD ( $=6$ each group). (A) BW; (B) FBG level; (C) HOMA-IR score; (D) ALT; (E) p-values for the interactive effects of HFD and Liraglutide treatment on BWs, FBG levels, HOMA-IR scores, ALT and AST were $0.055,0.001,0.005,0.185$ and 0.001 , respectively. ${ }^{*} p<0.05$ for comparison between saline and Liraglutide treatments. ${ }^{\#} p<0.05$ for comparison between NC and HFD groups.

Abbreviations: HOMA-IR, homeostasis model assessment of insulin resistance; ALT, alanine aminotransferase; AST, aspartame aminotransferase; BW, body weight; FBG, fasting blood glucose; HFD, high-fat diet; NC, the mice fed with a normal chow diet.

\section{InsR, IGF-IR, IRS2, PI3K p85, Akt and $\alpha-$ SMA mRNA expression levels in liver tissues}

As shown in Figure 5, the factorial analyses revealed that there were significant differences in InsR, IGF-1R, IRS2, PI3K, Akt and $\alpha$-SMA mRNA expression levels between NC and HFD groups of mice and between Liraglutidetreated and saline-treated mice. Compared with NC group, HFD mice exhibited significantly downregulated expression of InsR, IRS2, PI3K p85 and Akt mRNA $(p<0.05)$, and upregulated expression of $\alpha$-SMA $(p<0.05)$. However, after treatment with Liraglutide, InsR, IGF-1R, IRS2, PI3K p85 and Akt mRNA expression levels were increased $(p<0.05)$, and $\alpha$-SMA mRNA expression was decreased $(p<0.05)$.

\section{InsR+IGF-IR, IRS2, pPI3K p85/Pi3K p85, pAkt/Akt and $\alpha$-SMA protein expression levels in liver tissues}

The protein expression levels of InsR+IGF-1R, IRS2, pPI3K p85/PI3K p85, pAkt/Akt and $\alpha$-SMA are shown in Figure 6. There were significant differences in InsR +IGF-1R, IRS2, pPI3K p85/PI3K p85, pAkt/Akt and $\alpha-$ SMA protein expression levels between NC and HFD mice and between Liraglutide-treated and saline-treated mice. Compared with NC group, HFD group exhibited significantly downregulated protein expression of InsR +IGF-1R, IRS2, pPI3K p85/PI3K p85 and pAkt/Akt $(p<0.05)$, and upregulated expression of $\alpha$-SMA $(p<0.05)$. However, after treatment with Liraglutide, the levels of InsR+IGF-1R, IRS2, pPI3K p85/PI3K p85 and pAkt/Akt proteins were increased $(p<0.05)$, and $\alpha$-SMA mRNA protein was decreased $(p<0.05)$.

\section{Discussion}

In the current study, we found that treatment with Liraglutide for 10 weeks markedly improved metabolic parameters and alleviated NAFLD by using HFDinduced mouse model. Animal models have been used to investigate the pathophysiology of hepatic IR and liver steatosis. Compared with genetic models of NAFLD, HFD-induced NAFLD mouse models are extensively used because genetic models are more expensive and less readily available than HFD-induced models. ${ }^{21-23}$ In the current study, C57BL/6J mice, which were fed with HFD for 10 weeks, successfully developed IR and typical hepatic steatosis based upon HOMA-IR assessment and HE staining. After treatment with Liraglutide at a clinically relevant concentration for 10 weeks, marked beneficial effects were observed in our model mice. Interestingly, Liraglutide not only effectively ameliorated hyperglycemia and IR but also markedly alleviated hepatic steatosis. HE and Oil Red O staining of liver specimens from model mice treated with Liraglutide revealed improved lobular structure, with fewer lipid droplets within cells, compared with that in mice of saline-treated group. Therefore, our findings demonstrate that Liraglutide has beneficial effects on diabetes- and obesity-related NAFLD. These results are consistent with the previous reports. ${ }^{24,25}$ The pharmacological mechanism underlying the effects of Liraglutide on NAFLD is not fully understood, but Liraglutide-mediated IR inhibition has been reported to be partially responsible for the protective effect of Liraglutide against NAFLD. ${ }^{26}$ Hepatic steatosis and IR are correlated, and decreased hepatic fat accumulation is associated with improved insulin sensitivity. ${ }^{27}$ We showed herein that Liraglutide intervention significantly lowered the increased FBG levels, 


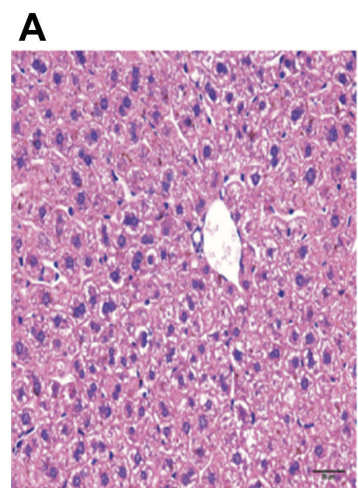

E

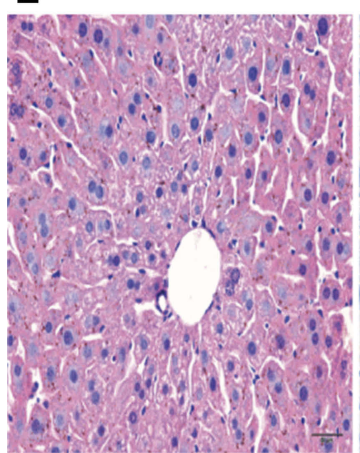

I

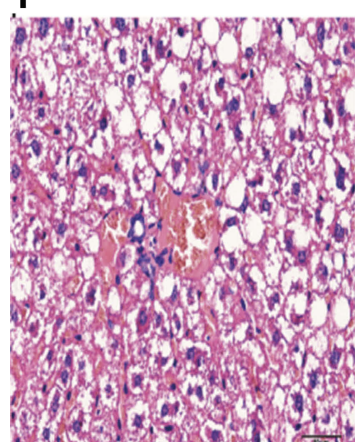

M

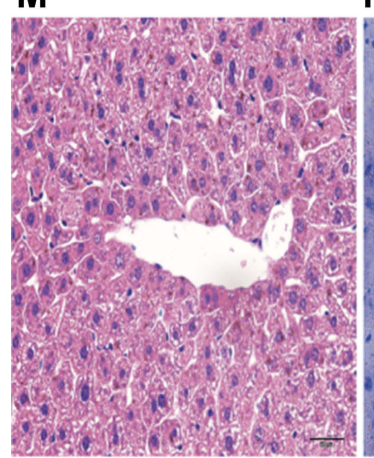

B

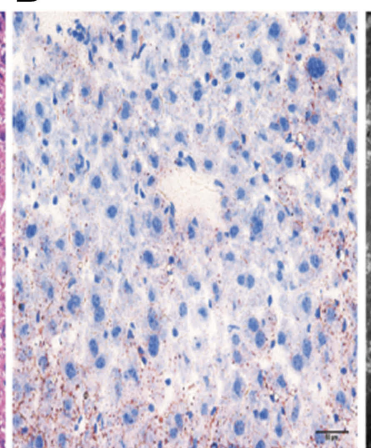

F

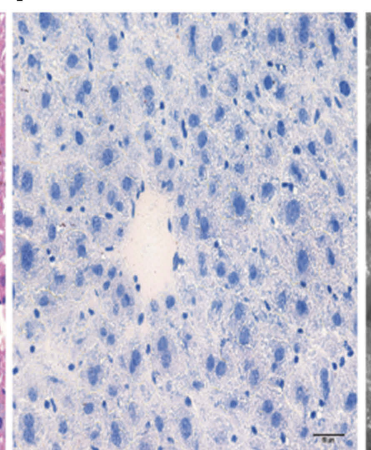

J

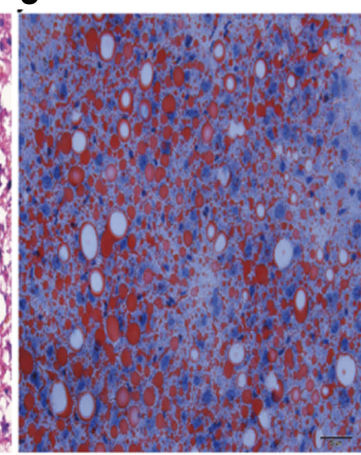

\begin{abstract}
N
\end{abstract}

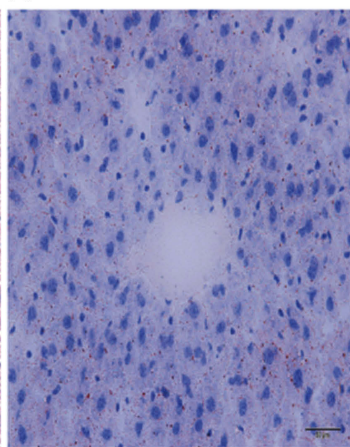

C

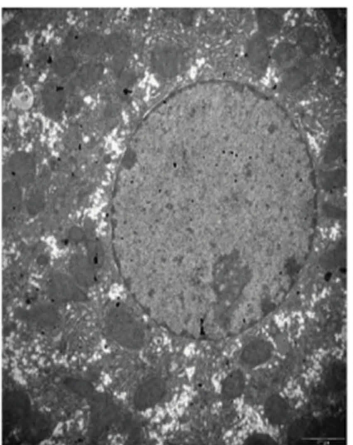

G

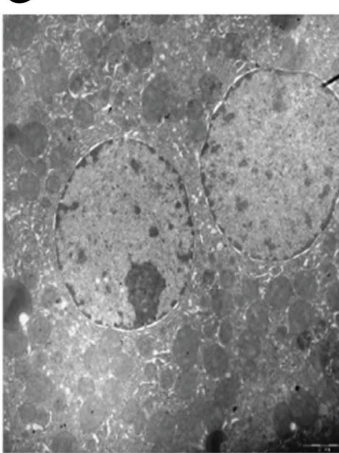

K

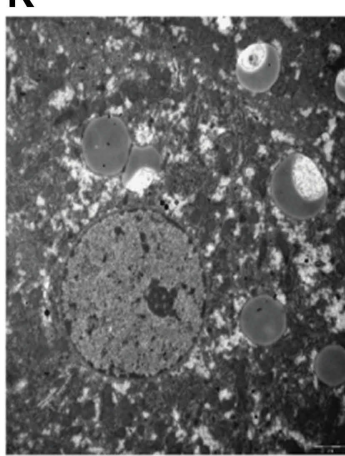

O

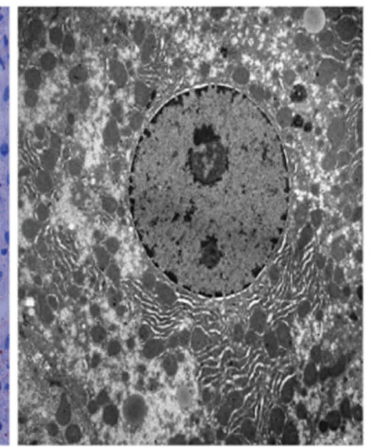

D

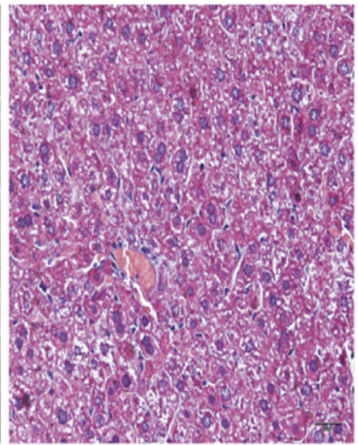

H

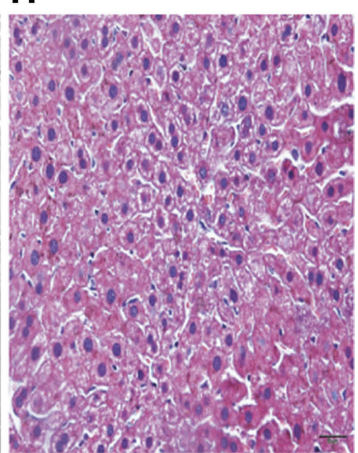

L

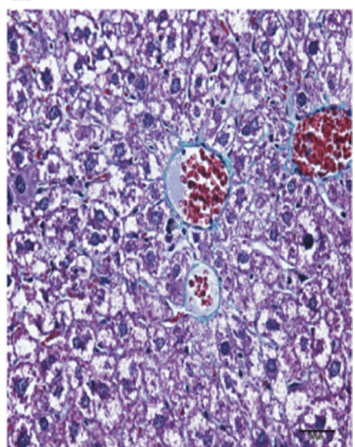

P

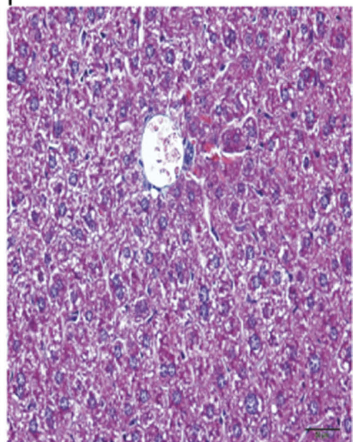

Figure 4 Histological evaluation of liver tissues with HE, Oil Red O staining, Mosson staining and transmission electron microscopy. Histological evaluation of liver tissues in each group stained with HE ( $\times 400)$, Oil Red $\mathrm{O}(\times 400)$ and Mosson staining $(\times 400)$. Ultrastructural changes in liver tissues from each group $(\times 10,000)$. ie, HE $(\mathbf{A}, \mathbf{E}, \mathbf{I}$ and $\mathbf{M})$; Oil Red O (B, F, J and $\mathbf{N}$ ); Mosson staining (D, H, L and $\mathbf{P})$; transmission electron microscopy (C, G, K and $\mathbf{O})$; $N+S$ group (A, B, C and $\mathbf{D})$; $N+L$ group (E, F, G and $\mathbf{H})$; $\mathrm{O}+\mathrm{S}$ group (I, J, $\mathbf{K}$ and $\mathbf{L})$; $\mathrm{O}+\mathrm{L}$ group $(\mathbf{M}, \mathbf{N}, \mathbf{O}$ and $\mathbf{P})$.

Abbreviations: $\mathrm{HE}$, hematoxylin and eosin staining; $\mathrm{N}+\mathrm{S}$, a normal saline-treated group; $\mathrm{N}+\mathrm{L}$, a Liraglutide-treated group; $\mathrm{O}+\mathrm{S}$, a normal saline-treated group; $\mathrm{O}+\mathrm{L}$, a Liraglutide-treated group. 
A

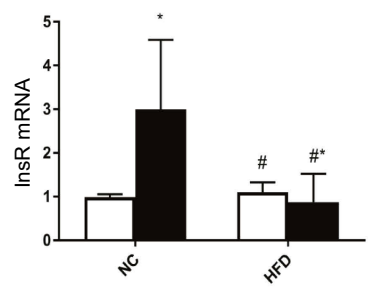

D

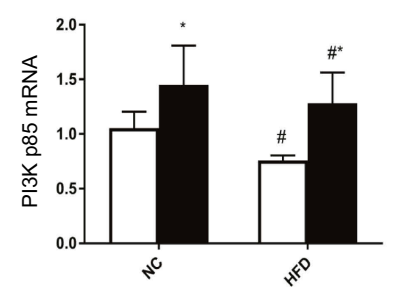

B

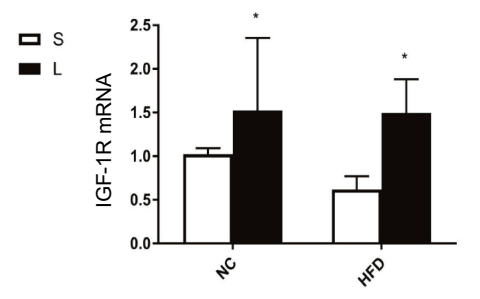

E

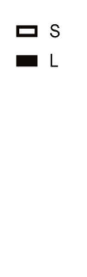

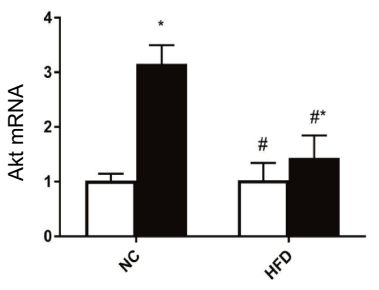

C

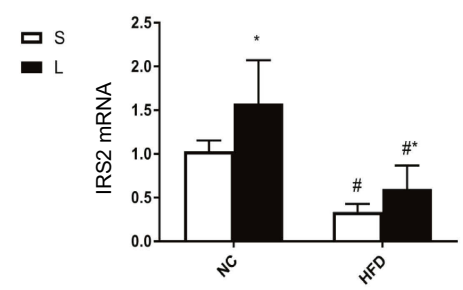

F

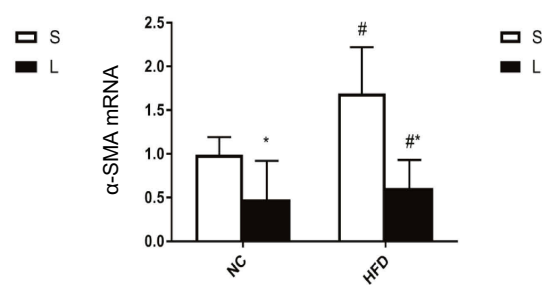

Figure 5 InsR, IGF-IR, IRS2, PI3K p85, Akt and $\boldsymbol{\alpha}$-SMA mRNA expression levels in mice after Liraglutide (L) or saline (S) treatment. Data were mean \pm SD ( $\mathrm{n}=6$ each group). $p$-values for the interactive effects of HFD and Liraglutide treatment on InsR, IGF-IR, IRS2, PI3K p85, Akt and $\alpha$-SMA mRNA expression levels were $0.05,0.338,0.253,0.53$, 0.001 and 0.087 , respectively. ie, InsR (A); IGF-IR (B); IRS2 (C); PI3K p85 (D); Akt (E); $\boldsymbol{\alpha}$-SMA (F). * $p<0.05$ for the comparison of the saline and Liraglutide groups; $\#$ $p<0.05$ for the comparison of NC and HFD groups.

Abbreviations: InsR, insulin receptor; IGF-IR, insulin-like growth factor-I receptor; IRS2, insulin receptor substrate 2; PI3K p85, PI3 kinase p85; Akt, protein kinase B; $\alpha$ -SMA, $\alpha$ - smooth muscle actin; HFD, high-fat diet; NC, the mice fed with a normal chow diet.

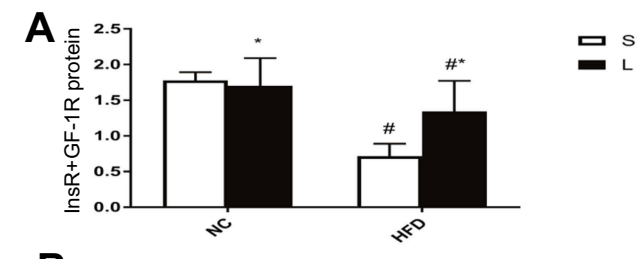

B

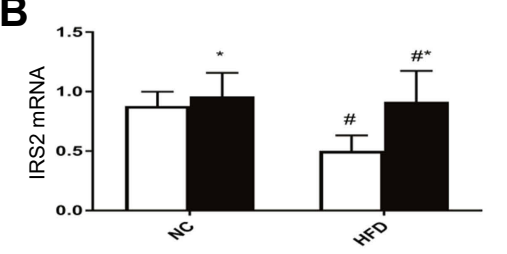

C
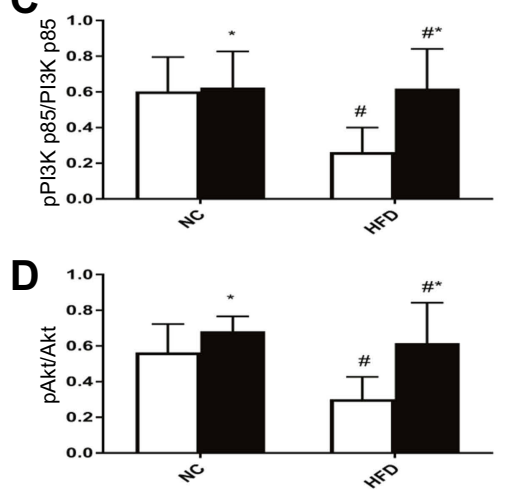

口 $\mathrm{s}$

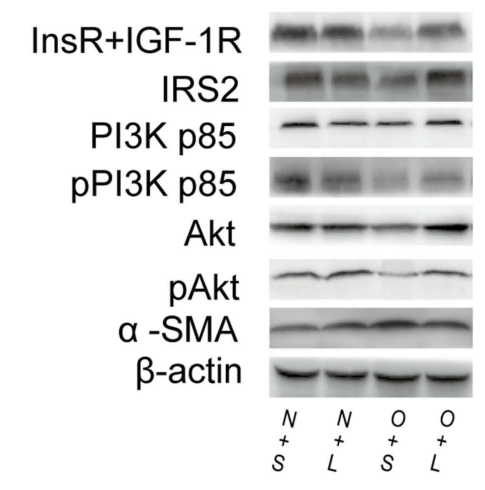

口 5

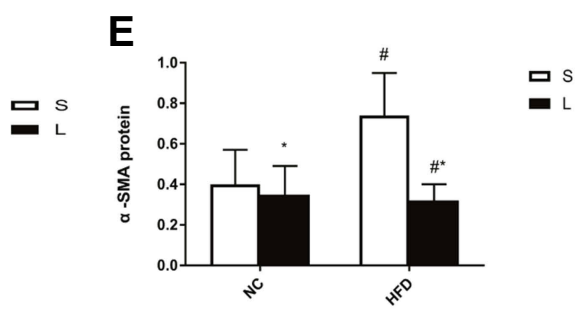

Figure 6 InsR+IGF-IR, IRS2, pPI3K p85/PI3K p85, p Akt/Akt and $\boldsymbol{\alpha}$-SMA levels in mice after Liraglutide (L) or saline (S) treatment. Data were mean \pm SD ( $\mathrm{n}=6$ each group). $p$-values for the interactive effects of HFD and Liraglutide treatment on InsR/IGF-IR, IRS2, pPI3K p85/PI3K p85, p Akt/Akt and $\alpha$-SMA levels were 0.01 I, 0.039, 0.044, 0.1 I 4 and 0.01 , respectively. ie, InsR/IGF-IR (A); IRS2 (B); pPI3K p85/PI3K p85 (C); p Akt/Akt (D); $\alpha$-SMA (E). *p<0.05 for the comparison of the saline and Liraglutide groups; ${ }^{\#}$ $p<0.05$ for the comparison of NC and HFD groups.

Abbreviations: InsR, insulin receptor; IGF-IR, insulin-like growth factor-I receptor; IRS2, insulin receptor substrate 2; PI3K p85, PI3 kinase p85; Akt, protein kinase B; $\alpha$ -SMA, $\alpha$ - smooth muscle actin; HFD, high-fat diet; NC, the mice fed with a normal chow diet. 
ameliorated hepatic steatosis and improved the HOMA-IR score in obese and diabesity mice, indicating that Liraglutide exerted an insulin-sensitizing effect. The liver expresses the GLP-1 receptor and regulates glucose metabolism, ${ }^{28}$ while several studies have shown that GLP-1 improves IR. ${ }^{29,30}$ However, the mechanism of GLP-1-mediated IR improvement still remains to be elucidated.

In the liver, insulin plays a key role in modulating glucose and lipid metabolism by suppressing gluconeogenesis/glycogenolysis and stimulating glycogen synthesis, lipogenesis and secretion of low-density lipoprotein in the fed state. Moreover, insulin plays a crucial role in hepatocyte regeneration and growth. ${ }^{31}$ Therefore, the integrity of the hepatic insulin signal transduction pathway plays a crucial role in the action of insulin in hepatocytes. Deficiency in the protein expression of any insulin signal transduction pathway components may induce hepatic IR. Furthermore, local hepatic IR may cause NAFLD. ${ }^{32}$ In the current study, HFD-induced NAFLD affected the expression of InsR/IGF-1R and IRS-2, indicating that the detrimental effects of HFD-induced NAFLD on the hepatic insulin signal transduction pathway occur at the pre-receptor levels. This phenomenon is the same as that seen in genetically obese animals. ${ }^{33}$ Tagawa et al showed a significant decrease in the level and activation of IRS-2 in a genetic model of fatty liver. ${ }^{34}$ Taken together, data from ours and other research groups suggest that the modification of early steps in insulin signaling plays an essential role in hepatic IR in NAFLD. Protein expression levels may be directly related to the phosphorylation and activation levels of that protein. IRS-2 proteins play an important role in transmitting signals from the InsR/IGF-1R to the PI3K/Akt kinases in hepatic insulin signaling. ${ }^{35}$ In hepatocytes, IRS-2 plays a major role in suppressing gluconeogenesis and regulating the PI3K/Akt cascade. ${ }^{14}$ In our experiments, the expression level of the IRS-2 protein, the $\mathrm{pPI} 3 \mathrm{~K} / \mathrm{PI} 3 \mathrm{~K}$ ratio and the $\mathrm{pAkt} / \mathrm{Akt}$ ratio were significantly decreased in the HFD-induced NAFLD model, indicating that the HFD-induced NAFLD model produces detrimental effects on IRS2/PI3K/Akt protein expression in hepatocytes similar to those of genetic models. ${ }^{35}$ We found that the HFDinduced inhibition of hepatic InsR/IGF-1R expression and the downregulation of IRS-2/PI3K/Akt signaling were reversed by Liraglutide treatment for 10 weeks. Interestingly, the HFD treatment downregulated InsR mRNA expression but not IGF-1R, suggesting that the effect of HFD-induced NAFLD on IRS2/PI3K/Akt signaling is mediated by InsR.
In conclusion, the current study provided evidence that Liraglutide decreased the FBG level, BW and HOMA-IR score in mice with NAFLD. Furthermore, Liraglutide improved hepatic steatosis, increased the InsR/IGF-1R and IRS2 expression levels and led to the activation of PI3K/Akt. and consequently alleviated hepatic steatosis by affecting the expression and activation of insulin signaling proteins.

\section{Conclusion}

The current study thereby provides evidence that Liraglutide ameliorates NAFLD and improves hepatic steatosis mainly by upregulation of the IRS2/PI3K/Akt signaling mediators.

\section{Acknowledgment}

This work was supported by the National Natural Science Foundation of China (81760146).

\section{Disclosure}

The authors report no conflicts of interest in this work.

\section{References}

1. Rinella ME. Nonalcoholic fatty liver disease: a systematic review. JAMA. 2015 Jun 9; 313(22): 2263-2273. doi:10.1001/jama.2015.5370

2. Arab JP, Barrera F, Gallego C, et al. High prevalence of undiagnosed liver cirrhosis and advanced fibrosis in type 2 diabetic patients. Ann Hepatol. 2016;15(5):721-728. doi:10.5604/16652681.1212434

3. Ballestri S, Zona S, Targher G, et al. Nonalcoholic fatty liver disease is associated with an almost twofold increased risk of incident type 2 diabetes and metabolic syndrome. Evidence from a systematic review and meta-analysis. $J$ Gastroenterol Hepatol. 2016;31(5):936-944. doi:10.1111/jgh. 13264

4. Smith BW, Adams LA. Nonalcoholic fatty liver disease and diabetes mellitus: pathogenesis and treatment. Nat Rev Endocrinol. 2011;7 (8):456-465. doi:10.1038/nrendo.2011.72

5. Porepa L, Ray JG, Sanchez-Romeu P, Booth GL. Newly diagnosed diabetes mellitus as a risk factor for serious liver disease. Cmaj. 2010;182(11):E526-31. doi:10.1503/cmaj.092144

6. Chen J, Zhao H, Ma X, et al. GLP-1/GLP-1R signaling in regulation of adipocyte differentiation and lipogenesis. Cell Physiol Biochem. 2017;42(3):1165-1176. doi:10.1159/000478872

7. He Q, Sha S, Sun L, Zhang J, Dong M. GLP-1 analogue improves hepatic lipid accumulation by inducing autophagy via AMPK/mTOR pathway. Biochem Biophys Res Commun. 2016;476(4):196-203. doi:10.1016/j.bbrc.2016.05.086

8. Wajcberg E, Amarah A. Liraglutide in the management of type 2 diabetes. Drug Des Devel Ther. 2010;4:279-290. doi:10.2147/DDDT.S10180

9. Samson SL, Bajaj M. Potential of incretin-based therapies for non-alcoholic fatty liver disease. J Diabetes Complications. 2013;27 (4):401-406. doi:10.1016/j.jdiacomp.2012.12.005

10. Sinaiko AR, Caprio S. Insulin resistance. J Pediatr. 2012;161 (1):11-15. doi:10.1016/j.jpeds.2012.01.012

11. Kotzka J, Muller-Wieland D. Sterol regulatory element-binding protein (SREBP)-1: gene regulatory target for insulin resistance? Expert Opin Ther Targets. 2004;8(2):141-149. doi:10.1517/14728222.8.2.141

12. Zheng F, Cai Y. Concurrent exercise improves insulin resistance and nonalcoholic fatty liver disease by upregulating PPAR-gamma and genes involved in the beta-oxidation of fatty acids in ApoE-KO mice fed a high-fat diet. Lipids Health Dis. 2019;18(1):6. doi:10.1186/ s12944-018-0933-z 
13. Guo S. Insulin signaling, resistance, and the metabolic syndrome: insights from mouse models into disease mechanisms. J Endocrinol. 2014;220(2):T1-T23. doi:10.1530/JOE-13-0327

14. Boucher J, Kleinridders A, Kahn CR. Insulin receptor signaling in normal and insulin-resistant states. Cold Spring Harb Perspect Biol. 2014;6(1). doi:10.1101/cshperspect.a009191

15. Roncero I, Alvarez E, Acosta C, et al. Insulin-receptor substrate-2 (irs-2) is required for maintaining glucokinase and glucokinase regulatory protein expression in mouse liver. PLoS One. 2013;8(4): e58797. doi:10.1371/journal.pone.0058797

16. Yoneyama Y, Lanzerstorfer P, Niwa H, et al. IRS-1 acts as an endocytic regulator of IGF-I receptor to facilitate sustained IGF signaling. eLife. 2018;7. doi:10.7554/eLife.42270

17. Kubota N, Tobe K, Terauchi Y, et al. Disruption of insulin receptor substrate 2 causes type 2 diabetes because of liver insulin resistance and lack of compensatory beta-cell hyperplasia. Diabetes. 2000;49 (11):1880-1889.

18. Talbot K. Brain insulin resistance in alzheimer's disease and its potential treatment with GLP-1 analogs. Neurodegener Dis Manag. 2014;4(1):31-40. doi:10.2217/nmt.13.73

19. Talbot K, Wang HY. The nature, significance, and glucagon-like peptide-1 analog treatment of brain insulin resistance in alzheimer's disease. Alzheimer's Dementia. 2014;10(1 Suppl):S12-25. doi:10.1016/j. jalz.2013.12.007

20. Liang Y, Li Z, Liang S, et al. Hepatic adenylate cyclase 3 is upregulated by liraglutide and subsequently plays a protective role in insulin resistance and obesity. Nutr Diabetes. 2016;6:e191. doi:10.1038/nutd.2015.37

21. Nagarajan P, Mahesh Kumar MJ, Venkatesan R, Majundar SS, Juyal RC. Genetically modified mouse models for the study of nonalcoholic fatty liver disease. World $J$ Gastroenterol. 2012;18 (11):1141-1153. doi:10.3748/wjg.v18.i11.1141

22. Arisqueta L, Navarro-Imaz H, Labiano I, Rueda Y, Fresnedo O. High fat diet overfeeding promotes non-detrimental liver steatosis in female mice. Am J Physiol Gastrointestinal Liver Physiol. 2018. doi:10.1152/ajpgi.00022.2018

23. Xu H, Zhou Y, Liu Y, et al. Metformin improves hepatic IRS2/PI3K/ Akt signaling in insulin-resistant rats of NASH and cirrhosis. J Endocrinol. 2016;229(2):133-144. doi:10.1530/JOE-15-0409

24. Ipsen DH, Rolin B, Rakipovski G, et al. Liraglutide decreases hepatic inflammation and injury in advanced lean non-alcoholic steatohepatitis. Basic Clin Pharmacol Toxicol. 2018;123(6):704-713. doi:10.1111/ bcpt.2018.123.issue-6
25. Zhu W, Feng PP, He K, Li SW, Gong JP. Liraglutide protects non-alcoholic fatty liver disease via inhibiting NLRP3 inflammasome activation in a mouse model induced by high-fat diet. Biochem Biophys Res Commun. 2018;505(2):523-529. doi:10. 1016/j.bbrc.2018.09.134

26. Tian F, Zheng Z, Zhang D, He S, Shen J. Efficacy of liraglutide in treating type 2 diabetes mellitus complicated with non-alcoholic fatty liver disease. Biosci Rep. 2018;38(6). doi:10.1042/ BSR20181304

27. Postic C, Girard J. Contribution of de novo fatty acid synthesis to hepatic steatosis and insulin resistance: lessons from genetically engineered mice. J Clin Invest. 2008;118(3):829-838. doi:10.1172/ JCI34275

28. Fan R, Kang Z, He L, Chan J, Xu G, Maedler K. Exendin-4 improves blood glucose control in both young and aging normal non-diabetic mice, possible contribution of beta cell independent effects. PLoS One. 2011;6(5):e20443. doi:10.1371/journal.pone. 0020443

29. Qin Y, Chen M, Yang Y, et al. Liraglutide improves hepatic insulin resistance via the canonical Wnt signaling pathway. Mol Med Rep. 2018;17(5):7372-7380. doi:10.3892/mmr.2018.8737

30. Cho YM, Fujita Y, Kieffer TJ. Glucagon-like peptide-1: glucose homeostasis and beyond. Annu Rev Physiol. 2014;76:535-559. doi:10.1146/annurev-physiol-021113-170315

31. Yaribeygi H, Farrokhi FR, Butler AE, Sahebkar A. Insulin resistance: review of the underlying molecular mechanisms. $J$ Cell Physiol. 2019 June;234(6):8152-8161. doi:10.1002/jcp.27603. Epub 2018 Oct 14.

32. Fatani S, Itua I, Clark P, Wong C, Naderali EK. The effects of diet-induced obesity on hepatocyte insulin signaling pathways and induction of non-alcoholic liver damage. Int $J$ Gen Med. 2011;4:211-219. doi:10.2147/IJGM.S17376

33. Friedman JE, Ishizuka $\mathrm{T}$, Liu $\mathrm{S}$, et al. Reduced insulin receptor signaling in the obese spontaneously hypertensive Koletsky rat. Am J Physiol. 1997;273(5 Pt 1):E1014-23.

34. Tagawa $\mathrm{H}$, Irie $\mathrm{J}$, Itoh $\mathrm{A}$, et al. Bile acid binding resin improves hepatic insulin sensitivity by reducing cholesterol but not triglyceride levels in the liver. Diabetes Res Clin Pract. 2015;109(1):85-94. doi:10.1016/j.diabres.2015.04.025

35. Balaji V, Pokrzywa W, Hoppe T. Ubiquitylation pathways in insulin signaling and organismal homeostasis. BioEssays. 2018;40(5): e1700223. doi:10.1002/bies.v40.5

Diabetes, Metabolic Syndrome and Obesity: Targets and Therapy

Dovepress

Publish your work in this journal

Diabetes, Metabolic Syndrome and Obesity: Targets and Therapy is an international, peer-reviewed open-access journal committed to the rapid publication of the latest laboratory and clinical findings in the fields of diabetes, metabolic syndrome and obesity research. Original research, review, case reports, hypothesis formation, expert opinion and commentaries are all considered for publication. The manuscript management system is completely online and includes a very quick and fair peer-review system, which is all easy to use. Visit http://www.dovepress.com/testimonials.php to read real quotes from published authors. 